\title{
Effects of an Angiotensin-Converting Enzyme Inhibitor, Alacepril, on Cardiovascular and Sympathetic Nervous Responses to Mental Stress in Patients with Essential Hypertension
}

\author{
Makoto SaItoh, Hiroyuki MiyaKoda*, Hideyuki KitamuRA*, \\ Toru Kinugawa*, Hiroshi Kotake* and Hiroto MashibA*
}

\begin{abstract}
The effects of an angiotensin-converting enzyme inhibitor, alacepril, on cardiovascular and plasma catecholamine responses to mental stress were studied. A mental arithmetic test (MAT) was carried out in 9 patients with essential hypertension before and after treatment with alacepril, $25 \mathrm{mg}$ once daily for 2 weeks, and in 9 age- and sex-matched normal subjects. Systolic blood pressure at rest and during MAT and the change in plasma norepinephrine concentrations during MAT were significantly greater in the hypertensive subjects than in the normal subjects. Alacepril significantly reduced systolic blood pressure, both at rest and during MAT, as compared to before treatment in the hypertensive subjects. Furthermore, alacepril significantly attenuated the change in plasma norepinephrine concentrations during MAT (from $127 \pm 76 \mathrm{pg} / \mathrm{ml}$ to $66 \pm 42 \mathrm{pg} / \mathrm{ml}$, $\mathbf{p}<0.05)$. These results suggest that alacepril significantly suppresses the augmented cardiovascular and sympathetic nervous responses to mental stress in patients with essential hypertension.

(Internal Medicine 32: 691-694, 1993)
\end{abstract}

Key words: mental arithmetic, plasma norepinephrine concentration, plasma epinephrine concentration

\section{Introduction}

Alacepril was developed in Japan as a novel angiotensinconverting enzyme (ACE) inhibitor characterized by long acting antihypertensive activity (1). Kinugawa and associates reported that alacepril significantly reduced blood pressure both at rest and during dynamic exercise in patients with essential hypertension (2). There have been no reports about the effects of alacepril on cardiovascular response to mental stress, which has been reported to be caused by increased sympathetic nervous activity (3-5). In this study, we examined cardiovascular and sympathetic nervous responses to mental stress in hypertensive subjects, and evaluated the effects of alacepril on the responsiveness.

\section{Methods}

The subjects were 9 untreated patients with mild-to-moderate essential hypertension and 9 age- and sex-matched normal subjects. The hypertensive subjects consisted of 3 men and 6 women ranging in age from 19 to 65 years $(51 \pm 15)$, and the normal subjects consisted of 3 men and 6 women without a history of hypertension, ranging in age from 20 to 70 (49 \pm 16$)$. Subjects with coronary artery disease, valvular heart disease, cardiomyopathy, and trained athletes were excluded from the study. All the hypertensive patients showed either systolic blood pressure (SBP) of greater than $160 \mathrm{mmHg}$ or diastolic blood pressure (DBP) ranging from $95 \mathrm{mmHg}$ to $115 \mathrm{mmHg}$ on at least two visits. None of the hypertensive subjects had been treated with anti-hypertensive medications. Those with congestive heart failure, moderate or severe retinopathy, or renal involvement were excluded.

The subjects arrived at the laboratory at 9:30 A.M. after a light breakfast, having avoided tea, coffee and cigarettes. There were no other dietary restrictions.

A 19-gauge catheter was placed in the antecubital vein of the right arm and a small amount of physiological saline was continuously infused. The subjects remained supine for 30

From the Department of Internal Medicine, Nishio Municipal Hospital, Nishio and *the First Department of Internal Medicine, Tottori University School of Medicine, Yonago

Received for publication January 20, 1993; Accepted for publication September 6, 1993

Reprint requests should be addressed to Dr. Makoto Saitoh, the Department of Internal Medicine, Nishio Municipal Hospital, 6 Kamiawara, Kumami-cho, Nishio 445 


\section{SAITOH et al}

minutes. Venous blood samples $(3 \mathrm{ml})$ were obtained to measure plasma catecholamine concentrations as an index of sympathetic nervous activity. Blood pressure was measured using an automatic sphygmomanometer (STBT-680, Nippon Colin, Komaki, Japan) on the left arm. Blood pressure and a 12-lead electrocardiogram were recorded. The subjects were asked to subtract, continuously, 7 from 1,013 as quickly as possible for 3 minutes. During this period, they were distracted by the rhythmic beats of a metronome. Blood pressure and the 12lead electrocardiogram were recorded during the last $30 \mathrm{sec}-$ onds of the mental arithmetic. A blood sample was obtained during the last 20 seconds of the mental arithmetic.

After 2 weeks of treatment with alacepril, $25 \mathrm{mg}$ once daily at 7 A.M., in the hypertensive subjects, the protocol was repeated at the same time of day as the first study.

The purpose and the nature of the protocol were explained to all subjects when they were recruited, and again on the morning of the study, and then informed consent was obtained. Permission for these investigations was granted by the Hospital Ethics Committee.

Blood samples were kept in ice water. After centrifugation, plasma samples were kept frozen at $-40^{\circ} \mathrm{C}$. Plasma norepinephrine (NE) and epinephrine (E) concentrations were measured by the THI (tri-hydroxyindole) method, with a high performance liquid chromatograph.

The results were expressed as mean \pm standard deviation. For statistical analysis, the paired t-test was used to compare data collected at rest with those collected during mental arithmetic and to compare the data collected from the hypertensive subjects before and after treatment. We used the unpaired t-test to compare data collected from the normal subjects with those from the hypertensive subjects. A p-value of less than 0.05 was taken to indicate a significant difference.

\section{Results}

Comparison of cardiovascular and sympathetic nervous responses to mental arithmetic between normal and hypertensive subjects.

In both groups, SBP, DBP, and heart rate (HR) were all significantly higher during mental arithmetic than at rest $(\mathrm{p}<0.01$, Table 1). SBP and DBP were significantly higher in the hypertensive subjects than in the normal subjects at rest and during mental arithmetic $(\mathrm{p}<0.01)$. There were no differences in HR between the two groups at rest and during mental arithmetic. The change in SBP was significantly greater in the hypertensive subjects than in the normal subjects during mental arithmetic $(\mathrm{p}<0.01)$. The changes in DBP and HR did not differ significantly between the two groups.

In both groups, plasma NE concentrations were significantly greater during mental arithmetic than at rest (normal subjects: $\mathrm{p}<0.05$; hypertensive subjects: $\mathrm{p}<0.01$ ). Plasma $\mathrm{E}$ concentrations were significantly greater during mental arithmetic than at rest only in the hypertensive subjects $(\mathrm{p}<0.01)$. Plasma NE and E concentrations did not significantly differ
Table 1. Cardiovascular and Sympathetic Nervous Responses to Mental Arithmetic in Normal and Hypertensive Subjects

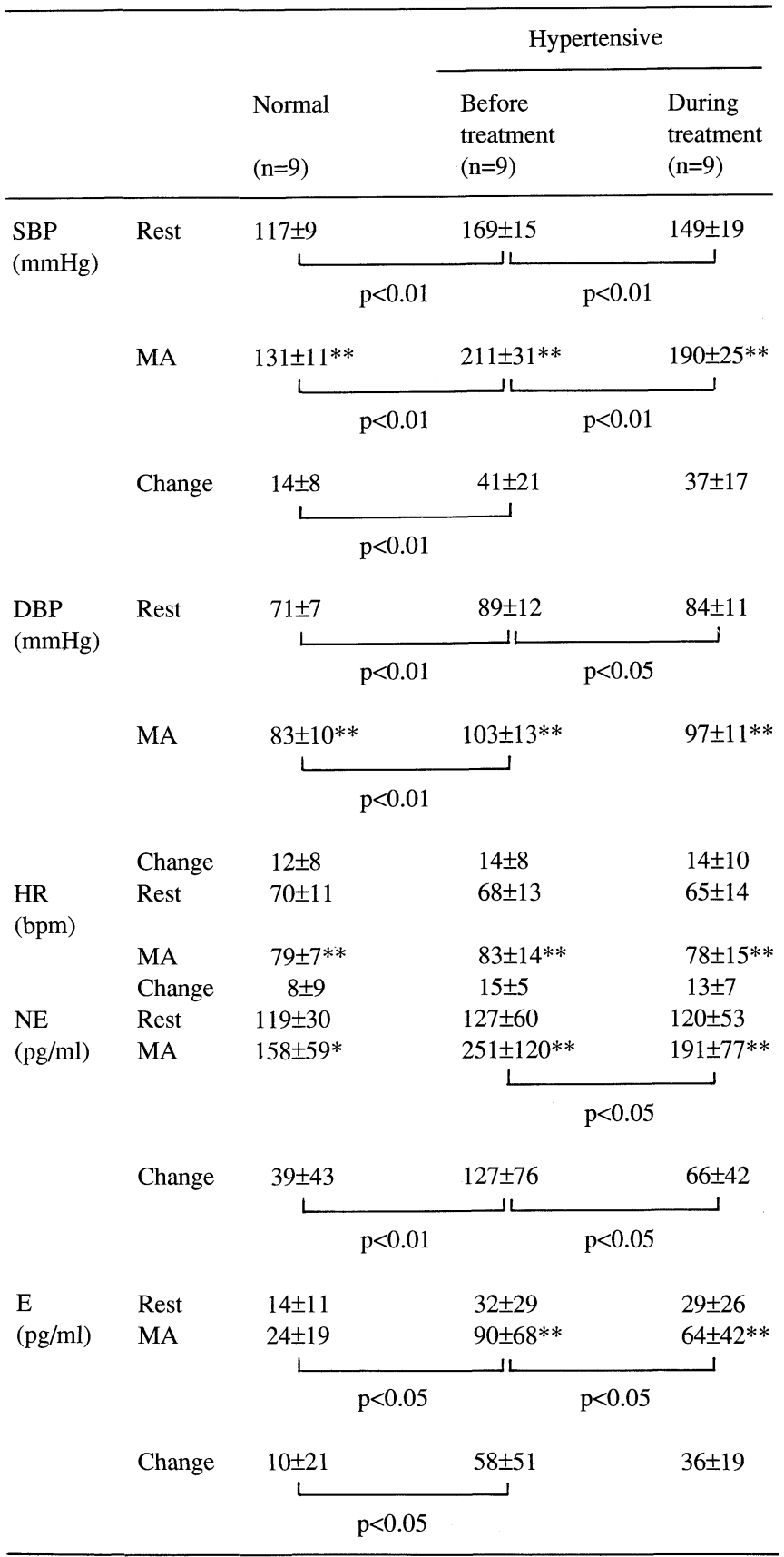

DBP: diastolic blood pressure, E: plasma epinephrine concentration. HR: heart rate, MA: mental arithmetic, NE: plasma norepinephrine concentration, SBP: systolic blood pressure. ${ }^{*} \mathrm{p}<0.05,{ }^{* *} \mathrm{p}<0.01$ vs. rest.

between the two groups at rest. Plasma E concentrations during mental arithmetic were significantly higher in the hypertensive subjects than in the normal subjects $(p<0.05)$. The changes in plasma $\mathrm{NE}$ and $\mathrm{E}$ concentrations induced by mental arithmetic were significantly greater in the hypertensive subjects than in the normal subjects $(\mathrm{p}<0.01$ for the former, $\mathrm{p}<0.05$ for the latter). 
The effects of alacepril on cardiovascular and sympathetic nervous responses to mental arithmetic in hypertensive subjects.

Although SBP and DBP at rest decreased significantly after treatment with alacepril $(p<0.01$ for the former, $p<0.05$ for the latter), HR at rest did not change. SBP during mental arithmetic decreased significantly after treatment with alacepril $(p<0.01)$, without significant change in DBP and HR.

The changes in SBP, DBP and HR during mental arithmetic were not affected by treatment with alacepril.

During treatment with alacepril, the plasma NE and E concentrations at rest remained unchanged. The plasma NE and $\mathrm{E}$ concentrations during mental arithmetic decreased significantly $(\mathrm{p}<0.05)$. The changes in plasma NE concentrations during mental arithmetic decreased significantly after treatment with alacepril $(\mathrm{p}<0.05)$.

\section{Discussion}

The impact of daily-life stress on the cardiovascular and sympathetic nervous system can be simulated and assessed in the laboratory by way of mental stress tests (3-13). In normal subjects, the effects of mental stress on plasma catecholamine responses are different from the effects of dynamic exercise (14). Dimsdale and Moss reported that E levels increased twofold during public speaking, whereas NE levels increased three-fold during physical exercise (14). We examined cardiovascular and sympathetic nervous responses to mental stress in hypertensive subjects.

Hemodynamic changes during mental arithmetic have been reported to be caused by an increased sympathetic outflow augmenting cardiac output and decreasing total peripheral vascular resistance (3-5). In the present study, both normal and hypertensive subjects showed significant increases in SBP, DBP, HR and plasma NE concentrations during mental arithmetic. Only the hypertensive subjects showed a significant increase in plasma $\mathrm{E}$ concentrations during mental arithmetic. Eliasson and associates reported that hypertensive subjects showed a significant increase in plasma NE and E concentrations during mental stress (20 minutes of color word test), but normal subjects showed no significant change in either plasma $\mathrm{NE}$ or E concentrations during the same test (4). It is unclear why, in the study by Eliasson and associates, the normal subjects showed no significant increase in plasma NE concentrations during mental stress.

In the present study, SBP at rest and during mental arithmetic, the increase in SBP induced by mental arithmetic, the plasma $\mathrm{E}$ concentrations during mental arithmetic and the change in the plasma NE and E concentrations caused by mental arithmetic were significantly greater in the hypertensive subjects than in the normal subjects. Thus, the hypertensive subjects were thought to show augmented cardiovascular and sympatho-adrenal responses to mental stress.

Although several studies have reported the effects of antihypertensive drugs on cardiovascular responses to mental stress (6-13), there have been few concerning the effects of antihypertensive drugs on both cardiovascular and sympathetic nervous responses to mental stress $(12,13)$. Eliasson and associates examined the effects of metoprolol, propranolol and hydrochlorothiazide on cardiovascular and sympatho-adrenal responses to mental stress (a color word test) in hypertensive patients $(12,13)$. In three groups, treatment significantly reduced blood pressures at rest and during mental stress compared to the values before treatment. However, treatment did not affect the plasma NE and $\mathrm{E}$ concentrations at rest and during mental stress compared to the values before treatment $(12,13)$.

In the present study, alacepril reduced SBP and DBP at rest, SBP during mental arithmetic, the plasma NE concentrations during mental arithmetic and the increase in plasma NE concentrations during mental arithmetic.

Miyajima and associates reported the close relation between the activated renin-angiotensin system and central outflow of sympathetic nervous activity in patients with renovascular hypertension by directly recording muscle sympathetic nervous activity (15). Furthermore, ACE inhibitors are thought to have an antihypertensive effect partly by decreasing the sympathetic nervous activity resulting from the inhibition of angiotensin II generation $(16,17)$.

Because the cardiovascular response to mental arithmetic is induced by activation of sympathetic nervous activity (3-5), the suppressive effect of alacepril on SBP response to mental arithmetic in the present study was thought to be induced by suppression of sympathetic nervous activity. Alacepril is absorbed from the digestive tract and metabolized into desacetylalacepril and captopril (18). Captopril has vasodilatory effects and inhibitory effects on sympathetic nervous activity by way of ACE inhibitory action with subsequent inhibition of angiotensin II generation (19). Minato and associates reported that desacetylalacepril itself has an inhibitory effect on peripheral sympathetic nervous activity without ACE inhibitory action (20). As we did not examine the response of the reninangiotensin system during mental arithmetic, it is unclear whether it was activated during mental arithmetic. We speculated that the inhibitory effects of alacepril on the plasma NE response during mental arithmetic may be due to both the direct inhibitory effect of desacetylalacepril and the putative indirect effect of captopril on peripheral sympathetic nervous activity.

Although alacepril significantly reduced SBP at rest and during mental arithmetic in the present study, HR at rest and during mental arithmetic did not change significantly after treatment with alacepril. To investigate the effects of an ACE inhibitor, ramipril, on autonomic nervous function, Sugimoto and associates performed measurements of heart rate variability in normal subjects, as indices of parasympathetic nervous tone, after a single oral administration of $5 \mathrm{mg}$ of ramipril or placebo (21). They reported that heart rate variability was significantly increased 120 minutes after administration of ramipril compared with placebo, and concluded that ramipril may enhance cardiac parasympathetic nervous activity (21). Besides the inhibitory effect on sympathetic nervous activity, 


\section{SAITOH et al}

the enhancing effect on parasympathetic nervous activity may in part be related to an absence of reflex tachycardia at rest and during mental arithmetic after treatment with alacepril in the present study.

As the present data may be limited by some factors, conclusions should be made with caution. This study was open in design and treated only a small number of subjects. We did not examine the performance rate during mental arithmetic in both normal and hypertensive subjects.

In conclusion, alacepril attenuates the augmented cardiovascular and sympatho-adrenal responses to mental stress seen in patients with essential hypertension. Whether this sympathoadrenal inhibition leads to a beneficial long-term effect in patients with essential hypertension is still uncertain, and further controlled studies with alacepril are required.

\section{References}

1) Kono T. Effects of a new angiotensin-converting enzyme inhibitor, 1(D-3-acetylthio-2-methylpropanoyl)-L-propyl-L-phenylalanine [DU1219], in normal men. Jpn Arch Int Med 30: 415, 1983.

2) Kinugawa $T$, Kitamura $H$, Ogino $K$, et ảl. Effect of alacepril on blood pressure and neurohumoral factors at rest and during dynamic exercise in patients with essential hypertension. Br J Clin Pharmacol 34: 366, 1992.

3) Brod J, Fencl V, Hejl Z, Jirka J. Circulatory changes underlying blood pressure elevation during acute emotional stress (mental arithmetic) in normotensive and hypertensive subjects. Clin Sci 18: 269, 1959.

4) Eliasson K, Hjemdahl P, Kahan T. Circulatory and sympatho-adrenal responses to stress in borderline and established hypertension. J Hypertens 1: 131, 1983.

5) Schmieder RE, Rueddel H, Neus H, Messerli FH, Von Eiff AW. Disparate hemodynamic responses to mental challenge after antihypertensive therapy with beta blockers and calcium entry blockers. Am J Med 82: $11,1987$.

6) Costa FV, Borghi C, Boschi S, Ambrosioni E. Cardiovascular response to mental stress: dynamic and isometric exercise in hypertensive patients treated with prizidilol hydrochloride (SK\&F 92657). Int J Clin Pharmacol Res 4: 373, 1984.

7) Floras JS, Hassan MO, Jones JV, Sleight P. Cardioselective and nonselective beta-adrenoceptor blocking drugs in hypertension: a comparison of their effect on blood pressure during mental and physical activity. $\mathbf{J}$ Am Coll Cardiol 6: 186, 1985.

8) Palombo C, Marabotti C, Genovesi-Ebert A, et al. Cardiovascular reac- tivity to physical and psychologic stress during long-term treatment with nitrendipine in essential hypertension. J Cardiovasc Pharmacol 12: S135, 1988.

9) Paran E, Neumann L, Cristal N, Lowenthal DT. Response to mental and physical stress before and during adrenoceptor blocker and angiotensinconverting enzyme inhibitor treatment in essential hypertension. Am J Cardiol 68: 1362, 1991.

10) Schmieder RE, Bähr $M$, Langewitz $W$, Rüddel $H$, Schächinger $H$, Schulte W. Efficacy of four antihypertensive drugs (clonidine, enarapril, nitrendipine, oxprenolol) on stress blood pressure. Am J Cardiol 63: 1333, 1989.

11) Rüddel H, Schmieder R, Langewitz W, Schulte W. Impact of antihypertensive therapy on blood pressure reactivity during mental stress. J Hum Hypertens 1: 259, 1988.

12) Eliasson K, Kahan T, Hylander B, Hjemdahl P. Responses to mental stress and physical provocations before and during long term treatment of hypertensive patients with $B$-adrenoceptor blockers or hydrochlorothiazide. Br J Clin Pharmacol 24: 1, 1987.

13) Eliasson K, Kahan T, Hylander B, Hjemdahl P. Reactivity to mental stress and cold provocating during long-term treatment with metoprolol, propranolol or hydrochlorothiazide. J Hypertens 4: S263, 1986.

14) Dimsdale JE, Moss J. Plasma catecholamines in stress and exercise. JAMA 243: 340, 1980.

15) Miyajima E, Yamada Y, Yoshida Y, et al. Muscle sympathetic nerve activity in renovascular hypertension and primary aldosteronism. Hypertension 17: 1057, 1992.

16) Cerasola G, Cottone $S$, D'Ignoto $G$, et al. Effects of enalapril maleate on blood pressure, renin-angiotensin-aldosterone. system, and peripheral sympathetic activity in essential hypertension. Clin Ther 9: 390, 1987.

17) Philipp T, Sharma AM, Thiede H-M, Kribben A. Sympathetic nervous activity and noradrenaline reactivity during angiotensin converting enzyme inhibition. Am J Cardiol 59: 55D, 1987.

18) Shionoiri H, Miyazaki N, Yasuda G, Miyakawa T, Takasaki I, Kaneko Y. Pharmacokinetics and antihypertensive effects of single and consecutive dosing of alacepril (DU-1219) in patients with severe hypertension. Curr Ther Res 38: 537, 1985.

19) Tsuda K, Shima H, Kuchii M, Nishio I, Masuyama Y. Effects of captopril on neurosecretion and vascular responsiveness in hypertension. Clin Exp Hyper-Theory Pract A9: 375, 1987.

20) Minato H, Hosoki K, Hayashi K, Sawayama T, Kadokawa T, Hashimoto M. Antihypertensive mechanism of alacepril. Effects of its metabolites on the peripheral sympathetic nervous system. Arzneim-Forsch/Drug Res 39: 319, 1989.

21) Sugimoto K, Kumagai $Y$, Tateishi T, Seguchi H, Ebihara A. Effects on autonomic function of a new angiotensin converting enzyme inhibitor, ramipril. J Cardiovasc Pharmacol 13: S40, 1989. 